The AstrophysicAL JouRnAL, 512:351-361, 1999 February 10

(C) 1999. The American Astronomical Society. All rights reserved. Printed in U.S.A.

\title{
THE LAST GASPS OF VY CANIS MAJORIS: APERTURE SYNTHESIS AND ADAPTIVE OPTICS IMAGERY
}

\author{
J. D. Monnier, ${ }^{1}$ P. G. Tuthill, ${ }^{1}$ B. Lopez,${ }^{2}$ P. Cruzalebes, ${ }^{3}$ W. C. Danchi, ${ }^{1}$ And C. A. Haniff ${ }^{4}$ \\ Received 1998 July 6; accepted 1998 September 17
}

\begin{abstract}
We present new observations of the red supergiant VY CMa at 1.25, 1.65, 2.26, 3.08, and $4.8 \mu \mathrm{m}$. Two complementary observational techniques were utilized: nonredundant aperture masking on the $10 \mathrm{~m}$ Keck I telescope, yielding images of the innermost regions at unprecedented resolution, and adaptive optics imaging on the ESO $3.6 \mathrm{~m}$ telescope at La Silla, attaining an extremely high $\left(\sim 10^{5}\right)$ peak-to-noise dynamic range over a wide field. For the first time the inner dust shell has been resolved in the nearinfrared to reveal a one-sided extension of circumstellar emission within $00^{\prime \prime}\left(\sim 15 R_{*}\right)$ of the star. The line-of-sight optical depths of the circumstellar dust shell at $1.65,2.26$, and $3.08 \mu \mathrm{m}$ have been estimated to be $1.86 \pm 0.42,0.85 \pm 0.20$, and $0.44 \pm 0.11$, respectively. These new results allow the bolometric luminosity of VY CMa to be estimated independent of the dust shell geometry, yielding $L_{*} \approx 2 \times 10^{5} L_{\odot}$. A variety of dust condensations, including a large scattering plume and a bow-shaped dust feature, were observed in the faint, extended nebula up to 4 " from the central source. While the origin of the nebulous plume remains uncertain, a geometrical model is developed assuming the plume is produced by radially driven dust grains forming at a rotating flow insertion point with a rotational period between 1200 and $4200 \mathrm{yr}$, which is perhaps the stellar rotational period or the orbital period of an unseen companion.

Subject headings: circumstellar matter - dust, extinction — stars: individual (VY Canis Majoris) infrared: stars - techniques: interferometric
\end{abstract}

\section{INTRODUCTION}

VY CMa (spectral type M5eIbp) is a very unusual star, displaying intense radio maser emission from a variety of molecules, strong dust emission in the mid-infrared, high polarization in the near-infrared, and large amplitude variability in the visible. Lada \& Reid (1978) estimated a distance of $\sim 1.5 \mathrm{kpc}$ for VY CMa by establishing its association with a known molecular cloud complex. Strong independent confirmation of this distance estimate has recently come from measuring the proper motions of long-lived $\mathrm{H}_{2} \mathrm{O}$ maser features in its outflow (Marvel 1996; Richards, Yates, \& Cohen 1998). This distance implies that VY CMa is also one of the most intrinsically luminous stars known in the Galaxy, $L_{*} \approx 5 \times 10^{5} L_{\odot}$ (Le Sidaner \& Le Bertre 1996). Such high luminosity is only expected from massive stars $\left(M_{*} \approx 25 M_{\odot}\right)$, and the extremely low effective temperature, $T_{*} \approx 2800 \mathrm{~K}$ (Le Sidaner \& Le Bertre 1996), indicates that $\mathrm{VY}$ CMa has $\sim 10^{4}$ yr remaining before exploding as a supernova (Brunish \& Truran 1982). Another sign of impending cataclysm is the extensive mass being lost by VY CMa into an optically thick circumstellar envelope. The mass loss rate for this star has been estimated using a variety of techniques (summarized in Danchi et al. 1994), yielding a consensus value of $\dot{M} \approx 2 \times 10^{4} M_{\odot} \mathrm{yr}^{-1}$.

Previous observations of the circumstellar envelope at a variety of wavelengths showed evidence of significant asymmetries. The dense molecular envelope surrounding the star supports strong maser emission of $\mathrm{SiO}, \mathrm{H}_{2} \mathrm{O}$, and $\mathrm{OH}$, showing spatial and redshift distributions that conform to

\footnotetext{
${ }^{1}$ Space Sciences Laboratory, University of California at Berkeley, Berkeley, CA 94720-7450.

${ }_{2}$ Observatoire de la Côte d'Azur, Departement Fresnel UMR 6528, BP 4229, F-06034 Nice Cedex 4, France.

${ }^{3}$ Observatoire de la Côte d'Azur, Departement Fresnel UMR 6528, Avenue Copernic, F-06130 Grasse, France.

${ }^{4}$ Mullard Radio Astronomy Observatory, Cavendish Laboratory, Madingley Road, Cambridge, CB3 OHE, England, UK.
}

no simple geometrical interpretation yet proposed (e.g., Benson \& Mutel 1979; Marvel 1996; Richards et al. 1998). Optical observers have noted VY CMa's peculiar one-sided nebulosity for much of the century. While these early observers described VY CMa as a binary or even multiple system (see Worley 1972 for some discussion), Herbig (1972), with the aid of polarizing filters, was able to show definitively that the apparent companions were simply light scattered from dust condensations in the nebula. Indeed, recent visible images from the Hubble Space Telescope (HST; Kastner \& Weintraub 1998) show a one-sided reflection nebula with a complicated arrangement of scattering features. High values of near-IR linear polarization further suggest asymmetries in the circumstellar emission at high spatial resolution, although no such asymmetries had been directly detected until this work. McCarthy (1979) reported asymmetry in the mid-IR emission of the dust shell, interpreting this as evidence for thermal emission from a disklike structure. Another important observational fact is that VY $\mathrm{CMa}$ is an irregular variable star showing 1-3 mag optical variation on the timescale of $\sim 2000$ days (Marvel 1996). Recent changes in the near-IR polarization direction (Maihara et al. 1976) and mid-IR spectrum (Monnier, Geballe, \& Danchi 1998) reveal that VY CMa is evolving on a variety of timescales.

We present new IR observations of VY CMa at 1.25, 1.65, $2.26,3.08$, and $4.8 \mu \mathrm{m}$. These data detail new aspects of the dust shell, from both scattered light and direct thermal dust emission, at unprecedented spatial resolution and dynamic range. After describing the novel observing techniques employed to make these images possible, we attempt to synthesize a coherent history of the VY CMa's recent massloss.

\section{OBSERVATIONS}

\subsection{Keck I Observations}

Aperture masking interferometry was performed by 

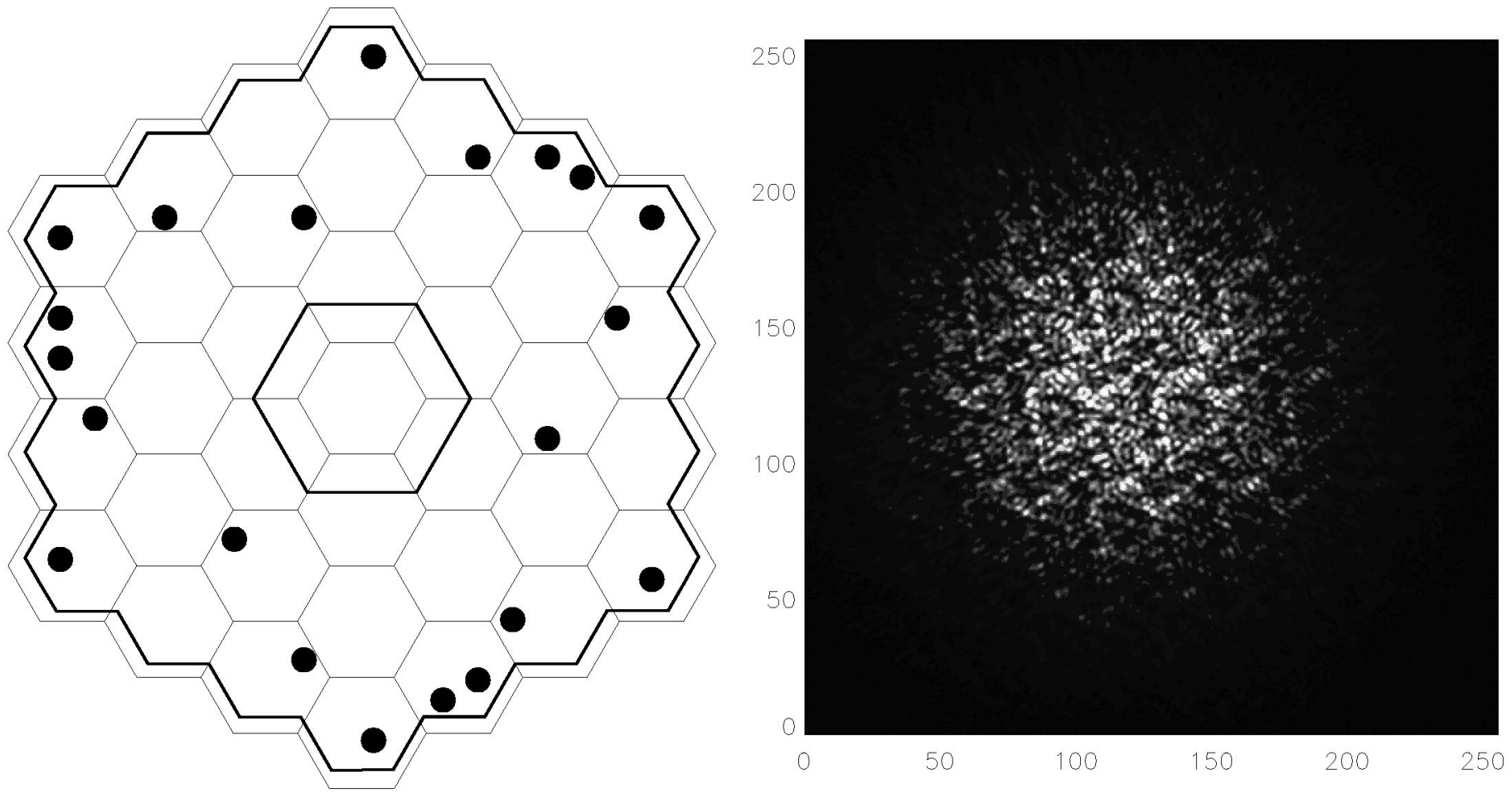

Fig. 1.-Pupil arrangement of a 21 hole, nonredundant aperture mask projected onto the hexagonal primary mirror of Keck I (left); single speckle frame observed while utilizing the aperture mask (right). The wavelength of observation was $3.08 \mu \mathrm{m}$, and the integration time was $0.149 \mathrm{~s}$. The horizontal and vertical axes are labeled in units of pixels, the plate scale being 20.3 mas pixel $^{-1}$.

placing custom-made plates in front of the Keck I secondary mirror, reducing light from the $10 \mathrm{~m}$ primary mirror to a predefined set of subapertures. An example of a 21 hole pupil arrangement and its corresponding interference pattern can be found in Figure 1, where each hole is roughly one atmospheric coherence patch in size $(35 \mathrm{~cm}$ at $\lambda=2.2$ $\mu \mathrm{m})$. The introduction of such a mask creates a series of overlapping two hole interference patterns projected onto the detector array, allowing the Fourier amplitude and phase of each baseline to be recovered without the addition of "redundancy" noise (e.g., Baldwin et al. 1986). By summing the atmospherically degraded Fourier phases around each closed triangle, we obtain the closure phase, an observable that is independent of the corrupting phase sheet above the subapertures (e.g., Jennison 1958). In the bright source limit, nonredundant aperture masking delivers a maximal signal-to-noise ratio of the closure phases, which is critical for reconstructions of noncentrosymmetric images. For fainter sources, a doubly redundant mask geometry in the shape of an annulus was used (Haniff \& Buscher 1992), balancing the desire for stellar flux with that for limited baseline redundancy. Further engineering and performance details may be found in Tuthill et al. (1999).

Raw visibilities were calibrated for the mean telescopeatmosphere transfer function by utilizing nearly contemporaneous observations of point-source stars. The observing regimen devoted an equal amount of time to such calibration stars, interleaved with the science targets under nearly identical conditions. Even so, seeing and other changes make the calibration process notoriously difficult, and mismatches between source and calibrator data introduced the greatest uncertainty into the final results.

Having obtained a set of calibrated visibilities and closure phases, there are a number of image reconstruction algorithms that minimize spurious artifacts, such as sidelobes arising from nonuniform or noisy sampling of the Fourier plane. One of the most popular of these, known as the "Maximum Entropy Method" (MEM; Gull \& Skilling 1984; Sivia 1987), has been used here. In order to check the reliability of the reconstructions, the MEM results have been compared with those from the CLEAN reconstruction algorithm (Högbom 1974; Cornwell \& Wilkinson 1981; Pearson \& Readhead 1984), which is also widely used in radio astronomy. Image orientation calibration and tests of the entire data analysis pipeline were performed by observing sets of known, close binary stars on each observing night.

VY CMa was observed in both January and December of 1997 at Keck I using the Near-Infrared Camera (NIRC), a cryogenically cooled $256 \times 256$ pixel InSb array (Matthews \& Soifer 1994). The magnified plate scale of 20.3 mas pixel $^{-1}$ was sufficient to Nyquist-sample fringes formed by the longest baselines at $2.2 \mu \mathrm{m}$; however, still finer fringes, formed at shorter wavelengths, were undersampled (Matthews et al. 1996). The dates, filter wavelengths, mask geometries, and integration times are detailed in Table 1. The unresolved star $\sigma \mathrm{CMa}$ (spectral type MOIab) was used for all Keck I calibration. Masking techniques only require $\sim 100$ speckle frames of the calibrator and source in order to produce high SNR maps of bright sources, such as VY CMa. Typical uncertainties in the closure phases obtained with nonredundant masks were $\pm 2^{\circ}$.

\subsection{ESO $3.6 \mathrm{~m}$ Observations}

Infrared observations of VY CMa were also obtained at the European Southern Observatory (ESO) $3.6 \mathrm{~m}$ telescope at La Silla, Chile, in 1996 December and 1997 January using the SHARPII+ and COMIC cameras. The 
TABLE 1

JOURNAL OF OBSERVATIONS

\begin{tabular}{|c|c|c|c|c|c|c|c|c|}
\hline & $\begin{array}{l}\text { Date } \\
\text { (UT) }\end{array}$ & $\begin{array}{c}\lambda \\
(\mu \mathrm{m})\end{array}$ & $\begin{array}{c}\Delta \lambda \\
(\mu \mathrm{m})\end{array}$ & $\begin{array}{l}T_{\text {int }} \\
(\mathrm{ms})\end{array}$ & Number of Frames & Telescope & Camera & Comments \\
\hline 1996 & Dec $29 \ldots \ldots$ & 1.253 & 0.296 & 100 & 480 & ESO $3.6 \mathrm{~m}$ & SHARPII + & Adaptive optics suboptimal \\
\hline 1997 & Jan $2 \ldots \ldots$ & 1.259 & 0.229 & 2000 & 200 & ESO $3.6 \mathrm{~m}$ & COMIC & With ADONIS adaptive optics system \\
\hline 1997 & Jan $2 \ldots \ldots$ & 4.832 & 0.590 & 10 & 700 & ESO $3.6 \mathrm{~m}$ & COMIC & With ADONIS adaptive optics system \\
\hline 1997 & Jan $30 \ldots \ldots$ & 1.647 & 0.018 & 134 & 100 & Keck I & NIRC & With 15 hole aperture mask \\
\hline 1997 & $\operatorname{Jan} 30 \ldots \ldots$ & 2.260 & 0.053 & 134 & 100 & Keck I & NIRC & With 15 hole aperture mask \\
\hline 1997 & Jan $30 \ldots \ldots$ & 3.083 & 0.101 & 134 & 100 & Keck I & NIRC & With 15 hole aperture mask \\
\hline 1997 & Dec $17 \ldots \ldots$ & 1.647 & 0.018 & 149 & 100 & Keck I & NIRC & With annulus aperture mask \\
\hline 1997 & Dec $19 \ldots \ldots$ & 1.647 & 0.018 & 149 & 100 & Keck I & NIRC & With annulus aperture mask \\
\hline 1997 & Dec $19 \ldots \ldots$ & 2.260 & 0.053 & 149 & 100 & Keck I & NIRC & With 21 hole aperture mask \\
\hline 1997 & Dec $19 \ldots \ldots$ & 3.083 & 0.101 & 149 & 100 & Keck I & NIRC & With 21 hole aperture mask \\
\hline
\end{tabular}

SHARPII + camera (Lacombe et al. 1995), which is sensitive from 1-2.5 $\mu \mathrm{m}$, contains a $256 \times 256$ pixel near-infrared camera and mulitobject spectrometer 3 detector array, and the high-resolution 35 mas pixel $^{-1}$ plate scale was utilized. The COMIC camera (Lacombe et al. 1995) has a wider wavelength coverage $(1.0-5.0 \mu \mathrm{m})$ and was used to followup the SHARPII + results and enable $5 \mu \mathrm{m}$ observing. However, this camera is equipped with only a $128 \times 128$ pixel format array, yielding a smaller field-of-view but maintaining the same high-resolution plate scale. Table 1 contains details of the observations.

VY CMa and point-source calibrator observations were interleaved to calibrate for changing seeing conditions and to fully characterize the faint wings of the telescope point spread function. $\omega \mathrm{CMa}$ (spectral type B2IV-Ve) was used as a calibrator source for both 1.25 and $4.8 \mu \mathrm{m}$ observations. The adaptive optics system was poorly adjusted while using the SHARPII + camera, resulting in uncertainty regarding the highest resolution image features.

The strength of the ESO system is the ability to obtain very high dynamic range images with nearly diffractionlimited resolution. The peak-to-noise ratio for the raw $J$-band images is $\sim 10^{5}$. A simple MEM deconvolution algorithm (Varosi \& Landsman 1995) was used to suppress the scattering halo from the bright central source; however, it is important to note that the prominent features discussed here were easily observed in the raw images, and the deconvolution process yielded only minor adjustments to the relative flux levels. In addition to the uncertainties in the adaptive optics closed-loop performance, the extraction of truly diffraction-limited images was hampered by the different spectral types of our source and calibrator targets, making broadband observations difficult to calibrate; however, the FWHM of the point-source calibrator images were always within $10 \%$ of those expected for a diffractionlimited $3.6 \mathrm{~m}$ telescope.

\section{RESULTS}

\subsection{Keck I Results}

Figure 2 shows image reconstructions at 1.65, 2.26, and $3.08 \mu \mathrm{m}$ from two epochs; observing details can be found in Table 1. Since VY CMa itself is completely unresolved by the Keck I telescope, we chose to smooth each image so that the FWHM of the central, dominant component is somewhat smaller than the Rayleigh criterion, $\Theta_{\mathrm{FWHM}}=$ $1.22(\lambda / D)$. The filled circle in each image frame represents the FWHM of the central component as displayed, 30 mas for the 1.65 and $2.26 \mu \mathrm{m}$ images and 40 mas for the $3.08 \mu \mathrm{m}$ observations. In addition to eliminating spurious features in the reconstructions as judged by internal consistency from multiple data sets, this process allows comparison of image contours generated from pupil masks with slightly different maximum resolutions. Image structures that appear at or below the lowest plotted contours are the result of systematic errors in the image reconstruction process, which is fundamentally limited by the calibration of the atmospheric transfer function.

The most dramatic feature in the high-resolution maps of Figure 2 is the one-sided emission in the near-IR. With the exception of a weak extension at $\lambda=3.08 \mu \mathrm{m}$, no circumstellar flux above $1 \%$ of the peak was detected to the north and northeast of the central source. Although the dust emission morphology is very similar at the three wavelengths, there are some departures at $1.65 \mu \mathrm{m}$, where the scattering efficiency of the grains is higher. Unfortunately, fluctuations in the atmospheric conditions limit the dynamic range in our final maps to $\sim 100$, preventing detection of low surface brightness features in the extended nebula.

The extended southern circumstellar emission is consistent with published near-IR polarization measurements. The 1-3 $\mu \mathrm{m}$ flux is polarized with a position angle between $60^{\circ}$ and $105^{\circ}$, with a trend toward higher angles for shorter wavelengths. Polarization angles and polarization strengths both appear to be slowly increasing in time with recent measurements giving linear polarizations of $7 \%$ at $1.65 \mu \mathrm{m}$ and $2 \%$ at $2.2 \mu \mathrm{m}$ (Forbes 1971; Maihara et al. 1976; Takami et al. 1992). Assuming the polarization arises from Rayleigh scattering, these observations suggest an asymmetric near-IR brightness distribution extending toward P.A. $170^{\circ}$ (or P.A. $-10^{\circ}$ ). This is in close agreement with the new high-resolution results presented in Figure 2.

The $10 \mathrm{~m}$ baselines afforded by the Keck I telescope allow us to discriminate stellar from nonstellar (extended) flux within our $5^{\prime \prime} \times 5^{\prime \prime}$ field of view. Fits to the high-resolution visibility data indicate the central source diameter is smaller than 20 mas; this is consistent with its bolometric luminosity and estimated effective temperature, which imply a stellar diameter between 10 and 16 mas (e.g., Danchi et al. 1994 and this paper). Extended emission (i.e., emission not originating from the unresolved central component) contributes $79.0 \% \pm 1.0 \%, 60.5 \% \pm 1.0 \%$, and $73.0 \% \pm 1.0 \%$ of the total flux at 3.08, 2.26, and $1.65 \mu \mathrm{m}$, respectively, appearing constant between January and December of 1997 to within uncertainties. Note how the fraction of the total flux arising from the extended component goes through a 


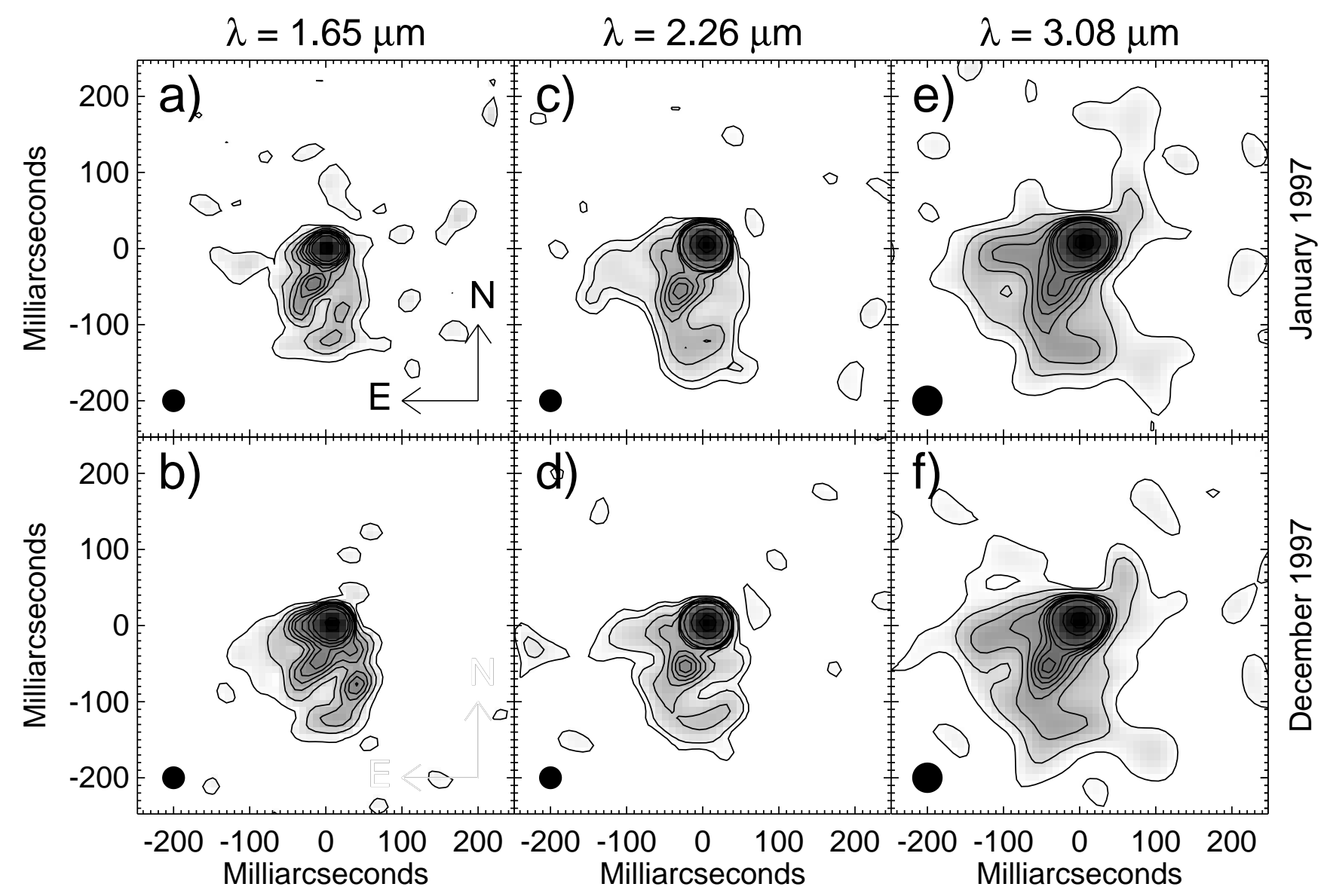

FIG. 2.- $(a-b)$ Image reconstructions of VY CMa observed at $1.65 \mu \mathrm{m}$ in 1997 January and December. The unresolved central source is displayed with a FWHM of 30 mas, as indicated by the "beam" circle in the lower left corner of each panel. The contour levels are $1.5 \%, 3.0 \%, 5.0 \%, 7.0 \%, 9.0 \%, 11.0 \%$, $15.0 \%, 20.0 \%, 30.0 \%$, and $80.0 \%$ of the peak. $(c-d)$ Image reconstructions of VY CMa observed at $2.26 \mu \mathrm{m}$ in 1997 January and December. The unresolved central source is displayed with a FWHM of 30 mas, while the contour levels are $0.7 \%, 1.0 \%, 2.0 \%, 3.0 \%, 4.0 \%, 5.0 \%, 6.0 \%, 10.0 \%, 30.0 \%$, and $70.0 \%$ of the peak. $(e-f)$ Image reconstructions of VY CMa observed at $3.08 \mu \mathrm{m}$ in 1997 January and December. The unresolved central source is displayed with a FWHM of 40 mas, while the contour levels are $1.0 \%, 2.0 \%, 4.0 \%, 6.0 \%, 8.0 \%, 10.0 \%, 12.0 \%, 14.0 \%, 20.0 \%, 50.0 \%$, and $80.0 \%$ of the peak.

minimum near $2.2 \mu \mathrm{m}$. This behavior is expected when the dust emission mechanism changes from being mostly thermal (in the red) to mostly scattering (in the blue).

The dynamic range of $\sim 100$ in the maps allows us to investigate the mass loss history of VY CMa for the last few hundred yr. The bright knot of emission at P.A. $155^{\circ}$ and separation $\sim 65$ mas appears in all the Keck I maps and is near the expected dust condensation radius, probably a clump of recently formed dust. This separation is slightly larger than the 50 mas dust shell inner radius from spherically symmetric models of the $11 \mu \mathrm{m}$ emission at maximum light (Danchi et al. 1994). The observed clump-star separation is a function of wavelength and is smaller for bluer wavelengths. In addition, the P.A. of the clump is larger for shorter wavelengths, which may also help explain the rotation of the polarization angle with wavelength discussed above. This behavior may arise from the differing wavelength dependencies for thermal emission and scattering of dust in the inner envelope. Because the clump-star separation is close to the diffraction limit, higher resolution images will be necessary to eliminate possible mapping ambiguities in the clump location. Lastly, we note in passing that the 2.26 and $3.08 \mu \mathrm{m}$ maps seem to exhibit a counterclockwise spiral structure.

\subsection{ESO $3.6 \mathrm{~m}$ Results}

3.2.1. $1.25 \mu \mathrm{m}$ Imagery
The ADONIS adaptive optics system and the infrared cameras on the ESO $3.6 \mathrm{~m}$ telescope allowed the recovery of nearly diffraction-limited (90 mas) images of a $6^{\prime \prime} \times 6^{\prime \prime}$ field around VY CMa with a peak-to-noise dynamic range of $\sim 10^{5}$. Dust structures in the outflow can be easily seen in scattered light at $1.25 \mu \mathrm{m}$, revealing a record of the massloss history of the last thousand yr.

Figure 3 shows a wide-field image of the circumstellar emission around VY CMa, and one can see a number of interesting structures. A bow-shaped scattering feature lies 4.2 to the south, with a brightness of approximately $0.003 \%$ of the peak flux. In addition, an arcing plume of emission extending west-northwest is observed, with a peak brightness of about $0.01 \%$ of the peak flux, terminating abruptly at 4".3. This is the first high-resolution image of the "curved nebulous tail toward $290^{\circ} "$ described by Herbig (1972). A similar sized field toward the east of VY CMa was also observed but revealed no nebulosity above $0.01 \%$ of the peak.

A bright knot of emission is also present $\sim 1^{\prime \prime}$ from the star at P.A. $220^{\circ}$. The peak emission from this southwest knot is $0.5 \%$ of the peak flux and is nearly 2 orders of magnitude brighter than other circumstellar features. This feature has been previously detected at $2.2 \mu \mathrm{m}$ by Cruzalebes et al. (1998) in 1994 September using the same telescope with the COME-ON + adaptive optics system. Other 


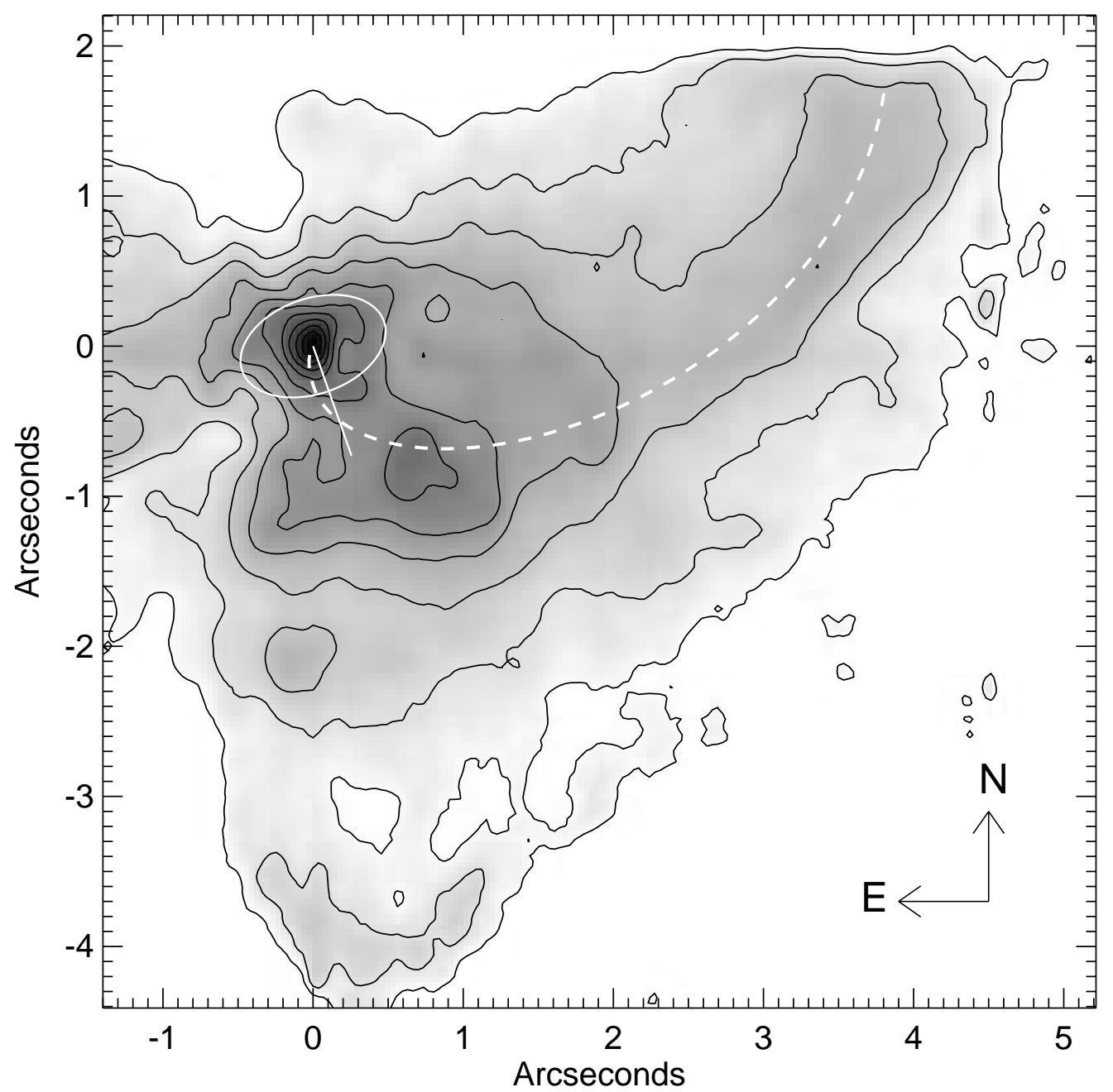

FIG. 3.-Adaptive optics image of VY CMa observed at $1.25 \mu \mathrm{m}$ in 1996 December using the SHARPII + camera. The overplotted solid and dashed white lines indicate the rotation geometry and outflow trajectory for the best-fit plume model discussed in $\S 4.4$. The contour levels are $0.001 \%$, $0.003 \%$, $0.010 \%, 0.032 \%, 0.10 \%, 0.316 \%, 1.0 \%, 3.16 \%, 10.0 \%$, and $31.6 \%$ of the peak.

weak features present include a ridge of emission extending from the star toward the southwest knot and a north-south partial shell of emission approximately 200 mas to the west of the central source.

A few detector artifacts must also be noted. The high brightness of this source caused the detector rows and columns sharing the central source region to show artificially high counts. Faint residual vertical and horizontal stripes can be seen extending from VY CMa caused by this effect. Additional spurious structure in the maps can be traced to diffraction spikes and scattering from various optical surfaces. Unfortunately, this scattering was not identical for the source and calibrator and has led to some low-level miscalibrations, such as some faint emission within an arcsecond of the source, which is especially obvious to the east and north (less than $0.01 \%$ of peak). There is probably a similar amount of faint residual flux to the west and south in the map, but it is not evident because of the bright dust features present.

Figure 4 shows VY CMa observed by the COMIC camera when the adaptive optics system was performing well. As can be seen, there is noticeably less emission observed within an arcsecond (especially to the east and north), while the other major features have remained largely unchanged. This image has a smaller field of view and has been included to show more detail of the nebula's inner region. The central source is partially resolved and slightly elongated north-south; however, a detailed fit to the size was not attempted because of uncertainties in the adaptive optics calibration at short wavelengths. As with the SHARPII + image in Figure 3, one can easily see the bright knot of emission to the southwest, and a partial shell of emission to the west. A recent $y$-band $(550 \mathrm{~nm})$ image of VY CMa taken by HST (Kastner \& Weintraub 1998) also shows evidence for a ridge of scattering material about 200 mas to the west; however, other features observed here (e.g., the bright southwest knot) do not appear. The HST images have a significantly lower peak-to-noise ratio $(\sim 500)$, which may explain their absence.

\subsection{2. $4.8 \mu \mathrm{m}$ Data}

The analysis of the $4.8 \mu \mathrm{m}$ COMIC data of VY CMa was hampered by poor background subtraction for the pointsource calibrator. Because low surface brightness features within $\sim 1^{\prime \prime}$ may be indicative of suboptimal adaptive optics performance as well as circumstellar dust emission, the point-source response of the entire telescope must be characterized before certain identification of dim, diffuse outflow condensations. Unfortunately, the calibrator chosen $(\omega$ $\mathrm{CMa}$ ) was not bright enough to allow high-quality back- 


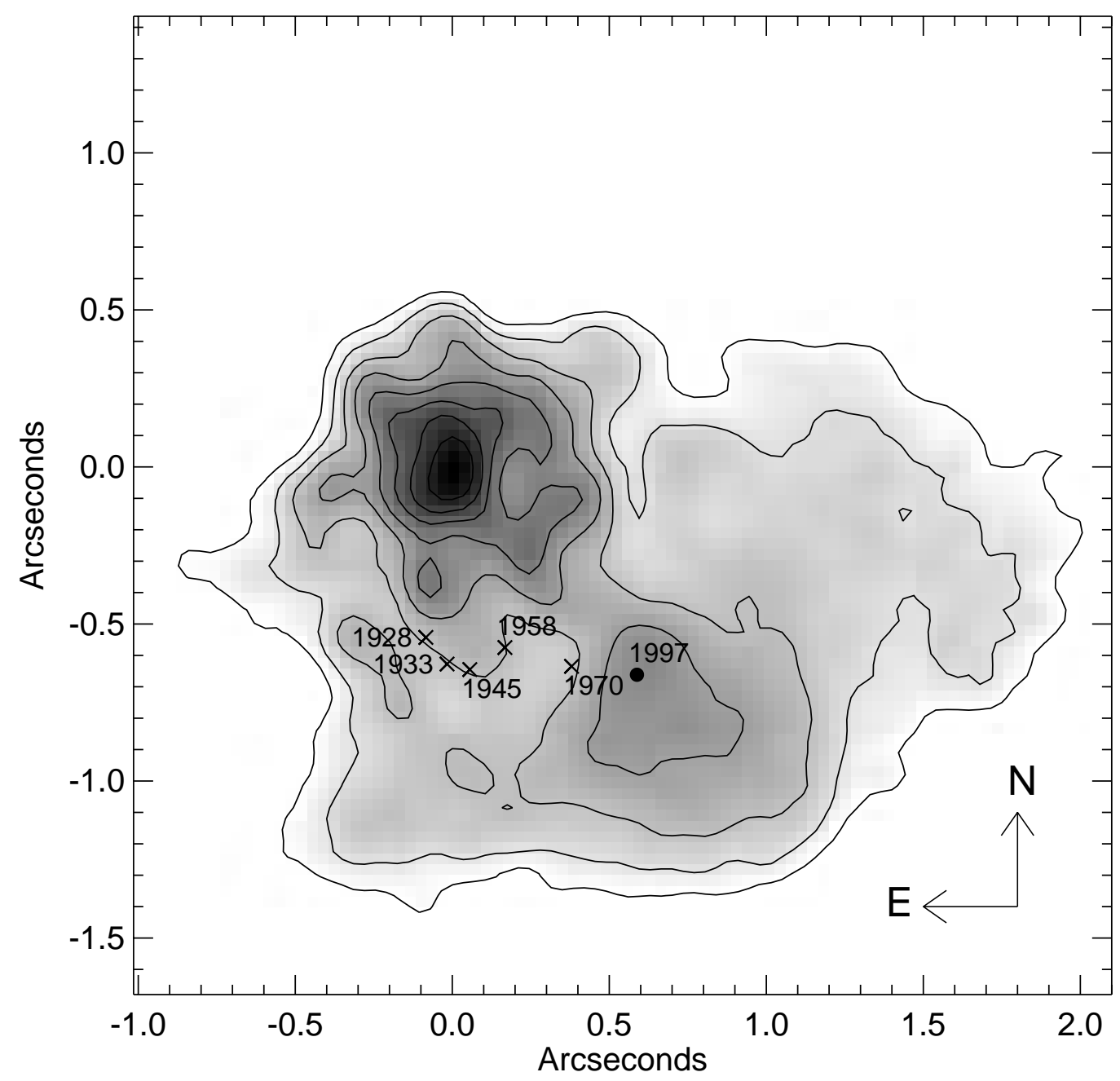

FIG. 4.-Adaptive optics image of VY CMa observed at $1.25 \mu \mathrm{m}$ in 1997 January using the COMIC camera. The cross symbols and accompanying dates represent the mean location of VY CMa's "companion" as observed in the visible by the U.S. Naval Observatory over the last 70 yr (see Wallerstein 1978 for a summary of these observations). The predicted position of the "companion" in 1997 has been extrapolated from the historical data and included as a filled circle. The contour levels are $0.010 \%, 0.032 \%, 0.10 \%, 0.316 \%, 1.0 \%, 3.16 \%, 10.0 \%$, and $31.6 \%$ of the peak.

ground subtraction. Hence, we feel that we can only report thermal structures at $1 \%$ of the peak with high confidence. The only such feature to report is a $\sim 1 \%$ brightness enhancement at $4.8 \mu \mathrm{m}$, which appears at the same location as the bright scattering knot seen at $1.25 \mu \mathrm{m}, \sim 1^{\prime \prime}$ to the southwest of the central source.

At $4.8 \mu \mathrm{m}$, the central source of VY CMa was partially resolved when compared with the point-source calibrator observed before and after. To investigate the highresolution morphology of the compact core of thermal emission, the data were analyzed utilizing the techniques of speckle interferometry. Frames were windowed to remove the relatively high background fluctuations, and then the mean power spectra were accumulated and averaged together over many exposures. Simple models of the brightness distribution were then fitted directly to the calibrated power spectra (or visibility data).

Drifts in the adaptive optics response precluded a meaningful measurement of thermal emission asymmetries in the compact emission core around VY CMa, but circularly symmetric fits to the visibility data do estimate the emission region's size. Uncertainty estimations were performed by analyzing various subsets of the 200 calibrator $\left(T_{\text {int }}=1.0 \mathrm{~s}\right.$ frame $\left.^{-1}\right)$ and 700 source frames $\left(T_{\text {int }}=0.01 \mathrm{~s} \mathrm{frame}^{-1}\right)$, and
Figure 5 shows the results for the full data set. Visibility data at short baselines are poorly calibrated because of seeing/adaptive optics changes and the different spectral types of the source and calibrator, a common artifact in speckle observations. Model fits were performed on data between $0.9 \operatorname{arcsec}^{-1}$ and $2.7 \operatorname{arcsec}^{-1}$, a range chosen to exclude low spatial frequency corruption due to source geometry, poor thermal background subtraction, low SNR data near the telescope cutoff frequency, and spectral type miscalibration. A single, circularly symmetric Gaussian brightness distribution was fitted (allowing the visibility at the origin to be a free parameter), yielding a FWHM of $163 \pm 8$ mas, which is marginally consistent with previously published $4.8 \mu \mathrm{m}$ results of $137 \pm 21$ mas by Dyck et al. (1984) and 152 mas by Bensammar et al. (1985). Possible systematic effects due to the different spectral types of the source and calibrator stars introduce another $\sim 10 \%$ uncertainty.

While the best-fit model shows systematic deviations from the data, the observations are too uncertain to justify the fitting of more complicated models. However, to emphasize the need for higher resolution and better calibrated observations, we note that the best-fit model for a Gaussian plus point source yields a radically different dust 


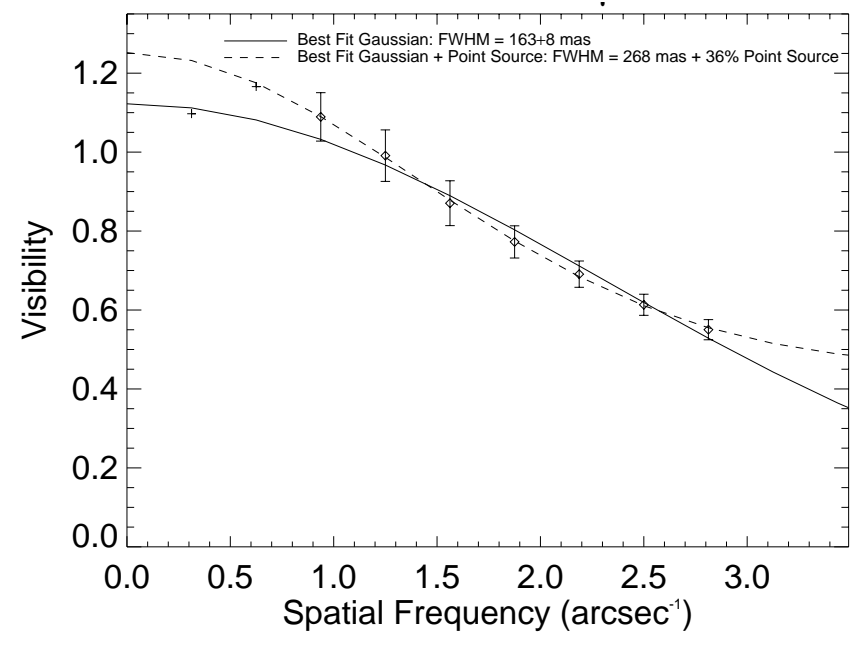

FIG. 5.-Azimuthally averaged visibility measurements of VY CMa at $4.8 \mu \mathrm{m}$ observed with the COMIC camera in 1997 January. The best-fit circularly symmetric Gaussian distribution, FWHM $163 \pm 8$ mas, is included on the figure as a solid line. The plus symbols are data points not used for the fitting. To illustrate the importance of acquiring longer baseline information, an alternative model consisting of a Gaussian distribution with FWHM 268 mas and a point source is shown as a dashed line.

shell geometry than the single Gaussian fit, with $36 \%$ of the flux in a point source surrounded by a larger Gaussian with FWHM 268 mas. Long baseline data at $4.8 \mu \mathrm{m}$ that resolves out the dust shell and measures the contribution of the unresolved component are necessary to constrain circumstellar models at this wavelength.

\section{DISCUSSION}

We proceed by dividing the wealth of structure observed in the circumstellar environment of VY CMa into size scales from the smallest (highest resolution) to most extended, discussing these in turn in the following subsections.

\subsection{Emission Asymmetry within $10 R_{*}$}

Based on classical spherical outflow models, stars such as VY CMa might be expected to be surrounded by limbbrightened shells when observed in the near-IR, where the optical depth is lower than in the visible. The highresolution images from the Keck I telescope (Fig. 2), however, bear no resemblance at all to this preconception but instead show emission to be one-sided, inhomogeneous, and highly asymmetric. Such structure may be interpreted in terms of one of three scenarios: less dust on the northern side of the nebula, high dust optical depth to the north of the star, or forward- scattering from the dust particles in a tilted equatorial disk.

If the dust shell is optically thin at near-IR wavelengths, then the lack of emission to the north implies less or cooler dust. Because northern dust would be in equal proximity to the star as the hot southern material, it seems unlikely that the dust could be cool. Furthermore, a dramatically rarified or evacuated region to the north also seems implausible when we consider the shape of the reflection nebula at bluer wavelengths. The almost total absence of scattered light to the north and northeast in the $1.25 \mu \mathrm{m}$ images in Figures 3 and 4 and also for images in the visible (Herbig 1972; Kastner \& Weintraub 1998) argues strongly that thick obscuration blocks the observer's line of sight into the northern region of the nebula. $\mathrm{H}_{2} \mathrm{O}$ masers present to the north and east of the VY CMa also indicate high dust densities in these locations (Richards et al. 1998; Marvel 1996).

Alternatively, we consider the case for an inner dust shell that is optically thick even at our reddest wavelength of 3.08 $\mu \mathrm{m}$. In this case, emission morphology traces lines-of-sight that can penetrate into hot, dusty regions before encountering unity optical depth. While spherically symmetric outflows would appear as centrally peaked shells, axisymmetric circumstellar density distributions can look quite different depending on the viewing angle (Efstathiou \& RowanRobinson 1990; Lopez, Mekarnia, \& Lefevre 1995). If the equatorial plane contains higher density material than that found near the poles, then the one-sided emission observed in the near-IR arises from warm dust in a polar region tilted somewhat south-southwest from our line of sight, a viewing angle consistent with the large-scale scattering asymmetry in the visible (the one-sided nebulosity). Furthermore, since the appearance of the object is determined to a great degree by optical depth effects, it is not surprising that the overall thermal emission extension occurs in roughly the same direction for $3.08 \mu \mathrm{m}$ as for the scattered light asymmetry observed at $1.65 \mu \mathrm{m}$. The only $10 \mu \mathrm{m}$ observations of VY CMa to detect asymmetry were by McCarthy (1979), who found an extension in the northeast/southwest direction. This is consistent with a disklike dust distribution of high optical depth with warm, evacuated polar regions, especially if large dust grains are present, enhancing mid-IR scattering.

As a third scenario, the forward-scattering of stellar light by dust contained in a tilted equatorial disk may contribute to the one-sided appearance of the nebula. If one assumes that the VY CMa dust envelope is, in a first approximation, a disklike structure with its south-west side tilted toward us, then the high surface brightness of the southwest side may be related to enhanced small-angle, forward-scattering by large dust particles. Such an effect has been observed in the circumstellar disk of UY Aur (Close et al. 1998), where the ratio between the bright side and faint one is about 10 . Although the ratio between the bright and faint lobes is larger than 100 for VY CMa, the presence of larger dust particles and a smaller disk inclination angle may help explain this difference.

The line-of-sight extinction due to the circumstellar shell and intervening interstellar dust can be estimated by using a combination of published infrared photometry, standard near-IR optical dust constants, and the high-resolution interferometric observations presented here. The interferometric data and photometry allow us to determine the absolute amount of flux at each wavelength that is coming from the star, separating out the circumstellar emission. By using an established extinction law (Ossenkopf, Henning, \& Mathis 1992) and published near-IR photometry of VY CMa near maximum light (Le Bertre 1993), the $2800 \mathrm{~K}$ blackbody spectrum of the star can be reddened until it matches the observed flux ratios of the star at 1.65, 2.26, and $3.08 \mu \mathrm{m}$. When using three wavelengths, this procedure may not have a solution, which would then imply the existence of errors in the flux measurements, the dust constants, or the effective stellar temperature. However, this procedure does yield a unique solution when applied to VY CMa, indicating that the set of initial assumptions are indeed selfconsistent. The line-of-sight optical depths (which includes the dust shell as well as interstellar extinction) for 1.65, 2.26, 
and $3.08 \mu \mathrm{m}$ are $2.1,1.0$, and 0.53 , respectively. The bolometric luminosity of VY CMa can also be estimated by dereddening the observed stellar flux without additional assumptions regarding the geometry of the dust shell. Assuming a distance of $1500 \mathrm{pc}$, this procedure yields $L_{*} \approx$ $1.7 \times 10^{5} L_{\odot}\left(T_{*} \approx 2800 \mathrm{~K} ; R_{*} \approx 5.5 \mathrm{mas} \approx 8.3 \mathrm{AU}\right)$. The uncertainties in these values are dominated by systematic uncertainties relating to the choice of dust constants and the effective temperature of the star. Calculations done using other dust constants (Draine \& Lee 1984; David \& Papoular 1990) and with effective temperatures as hot as $3500 \mathrm{~K}$, indicate an uncertainty of $\sim 20 \%$ in the values of the line-of-sight optical depths, and that the $L_{*}$ may be as high as $3.0 \times 10^{5} L_{\odot}$. This determination of $L_{*}$ is somewhat smaller (by factor of $\sim 2$ ) than the bolometric luminosity found for the spherical models by Le Sidaner \& Le Bertre (1996). However, spherical models of axisymmetric dust shells overestimate the bolometric luminosity when the viewing angle looks into less dense polar regions rather than through the optically thicker equatorial plane, and this may explain the discrepancy.

We must correct for interstellar extinction in order to estimate the optical depth of the circumstellar shell. Efstathiou \& Rowan-Robinson (1990) calculated various circumstellar dust shell models of VY CMa using interstellar extinction, $A_{V}$, from between 1.2 and 1.5. Despite the uncertainty in these estimates, we adopt $A_{V}=1.35$ and use the Mathis (1990) extinction law to correct the optical depth determinations for interstellar extinction. The line-of-sight optical depths of the circumstellar dust shell around VY $\mathrm{CMa}$ at $1.65,2.26$, and $3.08 \mu \mathrm{m}$ then become $1.86 \pm 0.42$, $0.85 \pm 0.20$, and $0.44 \pm 0.11$, respectively. These values are consistent with the hypothesis that the optical depth in the equatorial plane is roughly equal to or greater than unity at $3.08 \mu \mathrm{m}$, as long as the dust density is enhanced in an equatorial plane inclined to our line-of-sight.

High-resolution maps at our three infrared colors show similar structure; however, marked differences exist, particularly between the $1.65 \mu \mathrm{m}$ maps of Figures $2 a$ and $2 b$ and those of Figures $2 c-2 f$ further in the red. Such wavelengthdependent changes are the result of a number of physical phenomena. Regions of dust will yield varying spectral contributions depending on the local temperature, the importance of scattering, and the overall optical depth; all of these effects are strongly wavelength-dependent. Although we could make some attempt to disentangle these three effects with our multiwavelength maps, such an effort is probably more profitable in the context of the construction of a detailed, self-consistent radiative-transfer model of this star; this is left for future work.

While no relative motion of the dusty clumps was detected between January and December of 1997, follow-up high-resolution imaging of this source over the coming decades may show motion of individual clumps of emission, if they represent dust clouds being accelerated and driven away from the star. If the dusty clumps have an outflow velocity typical of the $\mathrm{OH}$ and $\mathrm{H}_{2} \mathrm{O}$ molecules $(\sim 33 \mathrm{~km}$ $\mathrm{s}^{-1}$ ), this 4.6 mas $\mathrm{yr}^{-1}$ outward motion could be detected with a temporal baseline of only a few yr.

\subsection{Southwestern Nebulosity and Bright Knot}

Turning our attention to the more extended structure revealed by adaptive optics, circumstellar emission at 1.25 , 2.2 , and $4.8 \mu \mathrm{m}$ within the inner $\operatorname{arcsec}$ (or $\sim 150 R_{*}$ ) of VY
CMa is dominated by the southwestern nebulosity and bright knot (see Fig. 4 and Cruzalebes et al. 1998). The direction of this extension is roughly perpendicular to the possible equatorial plane, as deduced from the one-sided reflection nebula seen in the visible. This near-IR, southwestern nebulosity supports the interpretation that light is preferentially escaping through less dense polar regions of a roughly axisymmetric dust distribution inclined toward the southwest. However, we note the position angle of the knot is $\sim 30^{\circ}$ different from the extension observed at high resolution in the inner dust shell at $1.65 \mu \mathrm{m}$ (see Figs. $2 a$ and $2 b$ ). An overall axisymmetric dust distribution with an axis lying roughly northeast/southwest would allow observations of dust emission only in the forward-facing, southwest polar lobe. Hence, dust inhomogeneities observed at higher resolution (see Fig. 2) may reflect the chaotic nature of local dust formation and may not be directly associated with the global symmetry of the dust shell.

The bright knot at $1^{\prime \prime}$ to the southwest may be partly due to an excess of stellar illumination through a hole in the inner dust shell. The relatively narrow line of emission connecting the knot with the central region lends some support for this conjecture; however, this may alternatively indicate a bridge of material extending from the star toward the bright knot. Figure 4 also summarizes a record of "binary companion" observations in the visible stretching back over $70 \mathrm{yr}$ (Wallerstein 1978). The current location of the southwest knot can be seen to lie along an extrapolated locus of these earlier points. Wallerstein (1978) attempted to explain this motion with a "rotating hole" hypothesis wherein the orbital motion of hole in the inner dust shell results in a moving "searchlight" beam. Rotation rates derived from this model are too high to be reconciled with single-star evolution of a red supergiant, implying the presence of a close binary companion or that VY CMa is actually a pre-main-sequence object still in possession of significant disk angular momentum.

Firm conclusions on the physical meanings of these features are difficult to draw mainly because of the many uncertainties of the dust shell geometry. For instance, the three-dimensional location of the southwest knot is very uncertain; hence the knot could represent scattering from a dust cloud existing above the pole or not far off the equatorial plane.

\subsection{Faint Dust Structures in the Outer Envelope}

Dust features were detected in scattered light at $1.25 \mu \mathrm{m}$ at distances up to $4^{\prime \prime}$ from the central source, which corresponds to a separation of $\sim 600 R_{*}$. The two main features observed at this size scale are the southern bow-shaped feature and the "curved, nebulous tail" stretching to the northwest in Figure 3.

The narrow arc- or bow-shaped feature to the south may have originated from a partial shell of dust formed during an asymmetric ejection event or, alternatively, as a cloud of higher velocity dust that was subsequently distorted by interactions with pre-existing circumstellar material. This dust material may be embedded in the expanding envelope around this star; if so, the dust cloud's velocity can be estimated from the expansion velocity of molecular gas $(\mathrm{OH}$, $\mathrm{H}_{2} \mathrm{O}$ ) in this region, 33-38 $\mathrm{km} \mathrm{s}^{-1}$ (Bowers, Johnston, \& Spencer 1983; Richards et al. 1998). This would date the time of ejection as $\sim 1000 \mathrm{yr}$ ago. 
In order to understand the origin of the dramatic western arc seen at $1.25 \mu \mathrm{m}$, we must consider two classes of explanations. The first class would not involve the actual transport (or collimated outflow) of dust in forming the arcing shape. This class of theories would hold that the circumstellar envelope has been severely disrupted, perhaps from vestigial pre-main-sequence material or the passage of a wide binary through the envelope. The nagging possibility that VY CMa is actually a pre-main-sequence object falls into this category (Lada \& Reid 1978; Kastner \& Weintraub 1998). Local obscuration and unusual illumination scenarios play a role here in shaping the appearance of the circumstellar environment. Until additional observations offer further support for such scenarios, we decline further speculation along these lines.

The second class of explanations involves the actual transport of dust in a collimated outflow. This idea is supported by the large contrast of the plume emission with respect to the surrounding nebulosity. The 1-2 orders of magnitude difference suggest that the density or albedo of the material along the plume is significantly higher. Assuming the plume material did indeed originate at the star and is embedded in the outflow, we further consider two cases depending whether the flow is "fast" or "slow." If the plume arises from a ballistic jet of high-velocity material ejected from the star, one would expect shock waves to form in the outflow. In order to investigate the ballistic jet hypothesis for the plume, a coronagraphic study was undertaken at Keck I in 1997 December to search for line emission. No emission above the continuum was observed in filters sensitive to the $\mathrm{H}_{2}(v=1-0)$ and $\mathrm{H}_{2}(v=2-1)$ lines, transitions indicative of shocked hydrogen. The coronagraphic study did confirm the blue spectrum of the plume (as expected from scattering from grains) by mapping the $[1.6 \mu \mathrm{m}] /[1.2 \mu \mathrm{m}]$ color.

On the other hand, if the dust in the plume has the "slow" outflow velocity typical of the $\mathrm{OH}$ and $\mathrm{H}_{2} \mathrm{O}$ the molecules, this 4.6 mas $\mathrm{yr}^{-1}$ radial motion implies that the plume process "turned on" roughly $1000 \mathrm{yr}$ ago. The long extension of the plume suggests that the ejection direction maintained itself, except for some apparent rotation, during most of the time since the beginning of the flow. We pursue this possibility below.

\subsection{Geometrical Model of Plume}

A simple geometrical model has been constructed to test the hypothesis that the plume represents radially driven dust grains produced at a rotating, or precessing, flow insertion point; for simplicity, we assume the plume lies in a plane. The velocity of the dust as a function of distance from the star has been based on the proper-motion study of the $\mathrm{H}_{2} \mathrm{O}$ masers by Richards et al. (1998). The dust is assumed to have an $8.5 \mathrm{~km} \mathrm{~s}^{-1}$ outflow velocity at $75 \mathrm{mas}$, increasing linearly to $33.0 \mathrm{~km} \mathrm{~s}^{-1}$ by 400 mas and staying constant thereafter, although our results are not critically dependent on the velocity law close to the star. We can now fit models based on three input parameters: the initial ejection direction, the period of rotation, and the inclination of the rotation axis (i.e., the viewing angle).

The model outflow was constrained to fit the outer ridge of the plume between $2^{\prime \prime} .5$ and 4 ..1 and to pass through the southwest knot located approximately $1^{\prime \prime}$ away from the star. The initial ejection direction was fitted to the direction of the near-IR curved extension seen toward the south in the Keck I images (Figs. $2 e$ and $2 f$ ). The best-fit plume model appears in Figure 3, which contains the shape of the outflowing plume structure as well as the projection of the rotation equator and the polar axis. The model fit indicates a rotational period between $1200-4200 \mathrm{yr}$, with an equatorial plane inclined $51^{\circ} \pm 5^{\circ}$ out of the plane of the sky and the north (or south) polar axis projected onto P.A. $200^{\circ} \pm 15^{\circ}$ with a $180^{\circ}$ ambiguity (i.e., the fit cannot tell us which lobe is facing toward the observer). With this geometry, the estimated time that the flow began is $950 \mathrm{yr}$ ago, and the flow insertion point is rotating clockwise when looked upon from the north pole. Unfortunately, the largest uncertainties in this fit are in the model assumptions themselves. The identification of the spiral-type morphology passing inward from the plume through the southwest knot and on in the Keck I images is highly speculative; hence our model parameters should be considered tentative.

In spite of such uncertainties, our simple empirical model shows a number of promising properties and furthermore serves as a useful starting point in exploring the details of the circumstellar morphology. The model is consistent with the assumption that the axisymmetry of the one-sided reflection nebula is due to an equatorial density enhancement with the equatorial plane inclined to the line-of-sight in such a way that scattering to the north and northeast is not seen. Efstathiou \& Rowan-Robinson (1990) constructed a radiative-transfer model of VY CMa based on a flareddisk geometry with an equatorial plane inclination angle of $\sim 47^{\circ}$. This is entirely consistent with the plume model developed above, although their model results depend greatly on the assumed density distribution of the disk. In addition, the VY CMa's derived rotational period of $\sim 2700$ $\mathrm{yr}$ is the right order of magnitude for evolution from an O-star progenitor (Heger 1998, private communication).

However, there is no obvious explanation for how the outflow could persist for $1000 \mathrm{yr}$. For instance, the evolutionary timescale for the convective elements within the photosphere has been found to be less than 1 yr (Tuthill, Haniff, \& Baldwin 1997; Wilson, Dhillon, \& Haniff 1997). However, this does not preclude the existence of longlasting surface inhomogeneities supported by magnetic fields, rotation, or some other complex phenomenon (e.g., Jupiter's Red Spot). If such a localized hot or cool spot exists, it may catalyze excess mass-loss or the creation of dust with a peculiar grain size distribution or chemical composition. The flow insertion point for this dust would be seen to turn at approximately the stellar rotation rate.

Alternatively, VY CMa may have experienced a dramatic stellar disruption about $1000 \mathrm{yr}$ ago that resulted in significant asymmetrical mass loss. This would link the origin of the southern bow-shaped dust feature with the beginning of the plume flow, since they are at the same distance from the central source. Such a disruption could occur if the outer surface layers become unstable to large-amplitude pulsations (Heger et al. 1997) or if the star undergoes an asymmetrical shell flash, although such internal, nova-like outbursts are not expected for stars more massive than $\sim 11 M_{\odot}$ (Iben 1998, private communication). This ejected material may persist in the inner envelope for years until the gas either disperses, falls back to rejoin the photosphere, or nucleates into dust grains and forms the arc. One problem with this picture is that a large quantity of gas would be required to form the high-contrast plume seen in the scattered light, the plume being at least 10-100 times brighter 
than its surrounding nebulosity. Such increased gas density in the upper atmosphere would cause a large pressure imbalance, spreading the gas around the star within $\sim 100$ yr (local sound speed is $\sim 3 \mathrm{~km} \mathrm{~s}^{-1}$ ). However, the large scattering contrast in the plume could also be caused by the presence of high-albedo dust grains instead of higher dust density. The scattering efficiency of grains can be dramatically increased by changing their size or their chemical composition (Papoular \& Pegourie 1983).

One last interpretation for the $2700 \mathrm{yr}$ period would be that it represents an orbital time period for a close binary companion. Between $31 \%$ and $62 \%$ of $\mathrm{O}$ stars (Garmany, Conti, \& Massey 1980; Stone 1981), progenitors of red supergiants, are found to be in binary systems. A low-mass companion located approximately 400 mas from VY CMa would have an orbital period of about $2700 \mathrm{yr}$, but it is unknown whether such a wind/binary interaction could produce the high-contrast plume observed.

\section{CONCLUSIONS}

We have reported new high angular resolution and high dynamic range observations of the circumstellar outflow of VY CMa. In addition to confirming previously observed features, several new structures were discovered. Diffraction-limited observations with the Keck I telescope allow the star and inner dust shell to be observed through the dusty envelope, revealing one-sided emission within 10 stellar radii. Combining these results with published photometry and existing optical dust constants, the line-of-sight optical depths of the circumstellar dust shell at 1.65, 2.26, and $3.08 \mu \mathrm{m}$ were estimated to be $1.86 \pm 0.42,0.85 \pm 0.20$, and $0.44 \pm 0.11$, respectively. In addition, these new results allow the bolometric luminosity of VY CMa to be estimated independent of dust shell geometry, yielding $L_{*} \approx$ $2 \times 10^{5} L_{\odot}$.

The adaptive optics system on the $3.6 \mathrm{~m}$ telescope has mapped the circumstellar envelope out to $\sim 4^{\prime \prime}$ from the faint stellar flux scattered off grains in the outflow. This combination of high spatial resolution ( $\sim 90$ mas) and high dynamic range $\left(\sim 10^{5}\right)$ was critical in resolving a highcontrast, scattering plume and a narrow, bow-shaped feature in the extended circumstellar environment.

The simplest density distribution consistent with the present data is an axisymmetric one with an equatorial density enhancement, the pole oriented roughly northeast/ southwest with the southwest lobe tilted toward us. This geometry attributes the lack of observed emission to the north and northeast to obscuration by dust near the equatorial plane. The clumpy near-IR emission on spatial scales of a few stellar diameters indicates the local mass-loss processes are strongly inhomogeneous. Although the present site of dust formation within $10 R_{*}$ (P.A. $\sim 155^{\circ}$ ) does not strictly conform to the overall geometry of the dust shell, time-averaged axisymmetry around a northeast/southwest axis is still possible and is most consistent with the full set of observations.

The curved, nebulous morphology of the dust plume extending to the northwest is consistent with radially driven dust grains formed at a rotating flow insertion point due to asymmetric mass-loss or a binary companion. A simple geometrical model indicates a rotational or orbital period of $\sim 2700 \mathrm{yr}$. The outer edge of the plume and the southern, bow-shaped feature lie approximately the same distance from the star suggesting a common origin in a stellar disruption $\sim 950 \mathrm{yr}$ ago. If such a disruption could liberate carbon-rich material from near the core, the long life of the plume and its high contrast with respect to the surrounding nebulosity might be explained as due to albedo variations of chemical origin.

The inner envelopes of late-type stars are proving much more complicated and exciting than expected. The clumpy scattering features in high-resolution HST and Keck I images; the nebulous curving plume and the southern, bowshaped feature embedded in the outflow; nonradial maser proper motions (Richards et al. 1998; Marvel 1996); the time evolution of the polarization direction (Maihara et al. 1976); and radical changes in mid-IR spectrum (Monnier et al. 1998) all testify to the presence of chaotic and violent dust formation processes around VY CMa, which occur on timescales of decades to millennia. Effects of stellar hot spot (Tuthill et al. 1997), high magnetic fields (McIntosh, Predmore, \& Patel 1994; Kemball \& Diamond 1997), rotation (Heger \& Langer 1998), large amplitude pulsations (Heger et al. 1997), and close binary companions (Morris 1980) may all play important roles in producing the thick circumstellar envelopes observed during the dying days of red supergiants and subsequently illuminated in the afterglow of supernova explosions.

We would like to thank Devinder Sivia for the maximumentropy mapping program "VLBMEM," which we have used to reconstruct diffraction-limited images. We wish to acknowledge the Keck Observatory personnel, especially Peter Gillingham, who have supported the aperture masking experiment and to whom we owe great thanks. J. D. M. would also like to thank Chris Matzner for helpful discussions. This research has made extensive use of the SIMBAD database, operated at CDS, Strasbourg, France, and NASA's Astrophysics Data System Abstract Service. Some of the data presented herein were obtained at the W. M. Keck Observatory, which is operated as a scientific partnership among Caltech, the University of California, and NASA. The Observatory was made possible by the generous financial support of the W. M. Keck Foundation. The results presented here have been partly based on observations collected at ESO, La Silla, Chile. C. A. H. is grateful to the Royal Society for continued financial support. This work is a part of a long-standing interferometry program at U. C. Berkeley, supported by the National Science Foundation (Grant AST-9315485 and AST-9731625) and by the Office of Naval Research (OCNR N00014-89-J-1583). In addition, scientific exchanges with the Observatoire de la Côte d'Azur were supported in part by a 1997 FranceBerkeley Fund grant to W. C. D. 


\section{REFERENCES}

Baldwin, J. E., Haniff, C. A., Mackay, C. D., \& Warnier, P. J. 1986, Nature, 320,595

Bensammar, S., Friedjung, M., Letourneur, N., \& Perrier, F. 1985, A\&A, 149, L1

Benson, J. M., \& Mutel, R. L. 1979, ApJ, 233, 199

Bowers, P. F., Johnston, K. J., \& Spencer, J. H. 1983, ApJ, 274, 733

Brunish, W. M., \& Truran, J. W. 1982, ApJ, 256, 247

Close, L. M., et al. 1998, ApJ, 499, 883

Cornwell, T. J., \& Wilkinson, P. N. 1981, MNRAS, 196, 1067

Cruzalebes, P., Lopez, B., Bester, M., Gendron, E., \& Sams, B. 1998, A\&A, 338,132

Danchi, W. C., Bester, M., Degiacomi, C. G., Greenhill, L. J., \& Townes, C. H. 1994, AJ, 107, 1469

David, P., \& Papoular, R. 1990, A\&A, 237, 425

Draine, B. T., \& Lee, H. M. 1984, ApJ, 285, 89

Dyck, H. M., Zuckerman, B., Leinert, C., \& Beckwith, S. 1984, ApJ, 287, 801

Efstathiou, A., \& Rowan-Robinson, M. 1990, MNRAS, 245, 275

Forbes, F. F. 1971, ApJ, 165, L83

Garmany, C. D., Conti, P. S., \& Massey, P. 1980, ApJ, 242, 1063

Gull, S. F., \& Skilling, J. 1984, Proc. IEEE 131, 6

Haniff, C. A., \& Buscher, D. F. 1992, J. Opt. Soc. Am., 9, 203

Heger, A., Jeannin, L., Langer, N., \& Baraffe, I. 1997, A\&A, 327, 224

Heger, A., \& Langer, N. 1998, A\&A, 334, 210

Herbig, G. H. 1972, ApJ, 172, 375

Högbom, J. 1974, ApJS, 15, 417

Jennison, R. C. 1958, MNRAS, 118, 276

Kastner, J. H., \& Weintraub, D. A. 1998, AJ, 115, 1592

Kemball, A. J., \& Diamond, P. J. 1997, ApJ, 481, L111

Lacombe, F., Marco, O., Eisenhauer, F., \& Hofmann, R. 1995, Messenger, 82,16

Lada, C. J., \& Reid, M. J. 1978, ApJ, 219, 95

Le Bertre, T. 1993, A\&AS, 97, 729
Le Sidaner, P., \& Le Bertre, T. 1996, A\&A, 314, 896

Lopez, B., Mekarnia, D., \& Lefebre, J. 1995, A\&A, 296, 752

Maihara, T., Noguchi, K., Oishi, M., Okuda, H., \& Sato, S. 1976, Nature, 259,465

Marvel, K. B. 1996, Ph.D. diss., New Mexico State Univ.

Mathis, J. S. 1990, ARA\&A, 28, 37

Matthews, K., Ghez, A. M., Weinberger, A. J., \& Neugebauer, G. 1996, PASP, 108, 615

Matthews, K., \& Soifer, B. T. 1994, Infrared Astronomy with Arrays: the Next Generation, ed. I. McLean (Dordrecht: Kluwer), 239

McCarthy, D. W., Jr. 1979, in IAU Colloq. 50, High Angular Resolution Stellar Interferometry, ed. J. Davis \& W.J. Tango (Sydney, Australia: Univ. Sydney Press)

McIntosh, G. C., Predmore, C. R., \& Patel, N. A. 1994, ApJ, 428, L29

Monnier, J. D., Geballe, T. R., \& Danchi, W. C. 1998, ApJ, 502, 833

Morris, M. 1980, ApJ, 249, 572

Ossenkopf, V., Henning, T., \& Mathis, J. S. 1992, A\&A, 261, 567

Papoular, R., \& Pegourie, B. 1983, A\&A, 128, 335

Pearson, T. J., \& Readhead, A. C. S. 1984, ARA\&A, 22, 97

Richards, A. M. S., Yates, J. A., \& Cohen, R. J. 1998, MNRAS, 299, 319

Sivia, D. S. 1987, Ph.D. diss., Cambridge Univ.

Stone, R. C. 1981, AJ, 86, 544

Takami, H., Shiba, H., Sato, S., Yamashita, T., \& Kobayahi, Y. 1992, PASP, 104, 949

Tuthill, P. G., Haniff, C. A., \& Baldwin, J. E. 1997, MNRAS, 285, 529

Tuthill, P., Monnier, J. D., Haniff, C. A., \& Danchi, W. C. 1999, in preparation

Varosi, F., \& Landsman, W. B. 1995, ASP Conf. Ser. 52, Astronomical Data Analysis Software and Systems II, ed. R. J. Hanisch, V. Brissenden, \& Jeannette Barnes (San Francisco: ASP), 515

Wallerstein, G. 1978, Observatory, 98, 224

Wilson, R. W., Dhillon, V. S., \& Haniff, C. A. 1997, MNRAS, 291, 819

Worley, C. E. 1972, ApJ, 175, L93 